University of Nebraska - Lincoln

DigitalCommons@University of Nebraska - Lincoln

Barry Chin Li Cheung Publications

Published Research - Department of Chemistry

March 2006

\title{
Fabrication of nanopillars by nanosphere lithography
}

\author{
Chin Li Cheung \\ University of Nebraska at Lincoln, ccheung2@unl.edu \\ R. J. Nikolic \\ Center for Micro and Nano Technology, Lawrence Livermore National Laboratory, Livermore, CA \\ C. E. Reinhardt \\ Center for Micro and Nano Technology, Lawrence Livermore National Laboratory, Livermore, CA \\ T. F. Wang \\ Directorate of Chemistry and Materials Sciences, Lawrence Livermore National Laboratory, Livermore, CA
}

Follow this and additional works at: https://digitalcommons.unl.edu/chemistrycheung

Part of the Chemistry Commons

Cheung, Chin Li; Nikolic, R. J.; Reinhardt, C. E.; and Wang, T. F., "Fabrication of nanopillars by nanosphere lithography" (2006). Barry Chin Li Cheung Publications. 4.

https://digitalcommons.unl.edu/chemistrycheung/4

This Article is brought to you for free and open access by the Published Research - Department of Chemistry at DigitalCommons@University of Nebraska - Lincoln. It has been accepted for inclusion in Barry Chin Li Cheung Publications by an authorized administrator of DigitalCommons@University of Nebraska - Lincoln. 


\title{
Fabrication of nanopillars by nanosphere lithography
}

\author{
C. L. Cheung ${ }^{1, *}$, R. J. Nikolić ${ }^{2}$, C. E. Reinhardt ${ }^{2}$, and T. F. Wang ${ }^{3}$ \\ ${ }^{1}$ Department of Chemistry and Center for Materials Research and Analysis, University of Nebraska-Lincoln, \\ Lincoln, NE 68588, USA \\ ${ }^{2}$ Center for Micro and Nano Technology, Lawrence Livermore National Laboratory, 7000 East Avenue, \\ Livermore, CA 94550, USA \\ ${ }^{3}$ Directorate of Chemistry and Materials Sciences, Lawrence Livermore National Laboratory, 7000 East Avenue, \\ Livermore, CA 94550, USA \\ * Correspondence: C. L. Cheung, email: ccheung2@unl.edu
}

\begin{abstract}
A low cost nanosphere lithography method for patterning and generation of semiconductor nanostructures provides a potential alternative to the conventional top-down fabrication techniques. Forests of silicon pillars of sub-500 nm diameter and with an aspect ratio up to 10 were fabricated using a combination of the nanosphere lithography and deep reactive ion etching techniques. The nanosphere etch mask coated silicon substrates were etched using oxygen plasma and a timemultiplexed "Bosch" process to produce nanopillars of different length, diameter and separation. Scanning electron microscopy data indicate that the silicon etch rates with the nanoscale etch masks decrease linearly with increasing aspect ratio of the resulting etch structures.
\end{abstract}

\section{Introduction}

Device physics is known to change when structures of materials are engineered at the nanoscale $[1,2]$. Nanospheres made of silica or organic polymer have been applied to generate various functional nanostructures, from photonic band gap materials [3] and surface plasmon sensor arrays [4] to nanometer-sized magnetic domains with orientation dependent magnetic anisotropy [5]. Nanosphere lithography (NSL) is an economical technique to generate single-layer hexagonally close packed or similar patterns of nanoscale features. Generally, NSL applies planar ordered arrays of nanometer-sized latex or silica spheres as lithography masks to fabricate nanoparticle arrays. NSL has been reported to generate nanowell arrays by conventional reactive ion etching $[6,7]$. Though NSL and reactive ion etching has been recently used to pattern nanometer-sized metal masks to produce columnar and spike structures [8-10], high aspect ratio pillar features with various separation spacing have not yet been demonstrated using this technique. Nanopillar patterned surfaces are usually made by deep ultraviolet lithography, electron beam lithography or nanoimprinting. These structures have been exploited for applications ranging from microfluidic chips for separation of long DNA molecules [11] and adhesion substrates for cell growth
[12] to the investigation of hydrophobicity and hydrophilicity on nanostructured surfaces [13].

Here we present the application of a low-cost NSL method to generate high aspect ratio silicon nanopillars by high density plasma reactive ion etching. The size and separation of these nanopillars were controlled by using polystyrene nanospheres of different sizes and etching these spheres with oxygen plasma to tailor the diameter of the nanosphere "lithography mask." Since these "masks" are densely packed with separation at the nanoscale, their packing density drastically affects the flow of reactive ion etchants and effluent ion species during the etching process. Thus, both the separation of etched "resists" and the etch time control the etch rates of silicon. An investigation of the as-fabricated structures with scanning electron microscopy (SEM) indicates that the etch rates of the masked silicon substrates decrease linearly with increasing aspect ratio of the resulting etched structures.

\section{Experiment}

Our strategy to fabricate the nanopillars consists of three major steps: spin coating of polystyrene beads on silicon, reactive ion etching (RIE) with oxygen to tailor the size of the polystyrene beads, and deep RIE of silicon to etch the pillar structures 


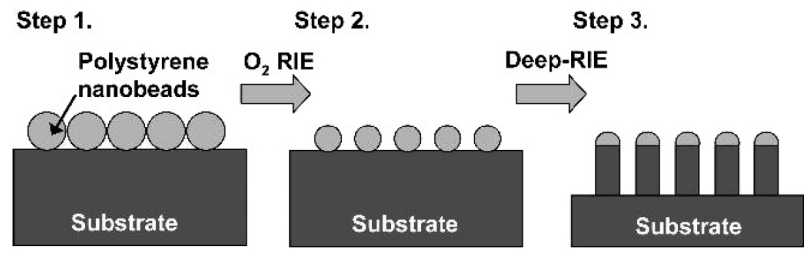

Figure 1. Schematic diagram of nanopillar fabrication by nanosphere lithography. Step 1: spin coat a hexagonally close packed monolayer of polystyrene beads on substrates. Step 2: tailor the size of the beads" "resist" by oxygen plasma etching. Step 3: etch the exposed semiconductor areas by deep reactive ion etching using the "Bosch" process.

(figure 1). For the NSL step, $2 \times 2 \mathrm{~cm}^{2}$ pieces of silicon or 2 inch silicon wafers (Prime grade, $2 \mathrm{~mm}$ thick, single and double sided polished silicon wafers, Recticon Enterprises, Inc., Pottstown, PA) were first cleaned by heating the samples in a $100 \mathrm{ml}$ RCA solution (an aqueous mixture of $30 \%$ hydrogen peroxide (Mallinckrodt Baker, Inc., Paris, KY), concentrated ammonium hydroxide (FisherScientific, Pittsburgh, PA), and DI water with the volume ratio of $1: 1: 5$.) at $70{ }^{\circ} \mathrm{C}$ for $30 \mathrm{~min}$, followed by a thorough rinse with DI water and drying in a stream of nitrogen. Then, a polystyrene bead solution prepared by a modified procedure in the literature [14] (50 $\mu$ l of Triton X-100 diluted with methanol by 1:400 (Sigma-Aldrich, Milwaukee, WI) and $350 \mu 1$ of polystyrene beads with diameter $500 \mathrm{~nm}$ (5050A, Duke Scientific, Palo Alto, CA)) was spin-coated on these samples with a commercial spin coater. The spin-coating program consists of three stages: (i) $400 \mathrm{rpm}$ for $10 \mathrm{~s}$ to spread the beads solution evenly; (ii) $800 \mathrm{rpm}$ for 2 min to spin away the excess bead solution; (iii) $1400 \mathrm{rpm}$ for $10 \mathrm{~s}$ to spin off the excess materials from the edges. The surfaces of the resulting substrates are strongly diffracted under room light to reflect a purple opal color. The diameters of the polystyrene bead coated substrates were then tailored by a parallel plate RIE etcher with $200 \mathrm{sccm}$ of oxygen and 8.4 sccm of tetrafluoromethane at a pressure of $200 \mathrm{mTorr}$ and radio frequency (RF) power of $100 \mathrm{~W}$ with various etch periods $(0,30,60,90,120$ s) (figure 2, row A).

Nanopillars were fabricated with the polystyrene bead coated silicon by applying the "Bosch" time-multiplexed RIE process $[15,16]$ in a commercial inductive coupled plasma etcher. Briefly, in the process, alternated cycles of etching in a flow of $\mathrm{SF}_{6}(12 \mathrm{sccm}, 12 \mathrm{~s})$ and passivation in a flow of $\mathrm{C}_{4} \mathrm{~F}_{8}$ $(85 \mathrm{sccm}, 9 \mathrm{~s})$ were used to etch the unprotected areas and to deposit fluorinated polymer to protect the side walls of the resulting etched structures. The plasma with these chemical species was generated with an RF power of $600 \mathrm{~W}$ and platen power of $14 \mathrm{~W}$ at a pressure of $4.5 \mathrm{mTorr}$. The temperature of the substrate was kept at $25{ }^{\circ} \mathrm{C}$ by cooling with a stream of helium. The samples were etched with different numbers of etch cycles and examined by scanning electron microscopy to investigate the relationship between the etch rate and aspect ratio of the etched structures.

\section{Results and discussion}

The three-step fabrication scheme has been successfully carried out to yield "forests" of silicon nanopillars of diame- ters from 250 to $350 \mathrm{~nm}$. After the NSL in step 1, SEM data showed that the spin-coated beads were packed mostly as monolayers of hexagonally closed packed (HCP) domains of 5-150 $\mu \mathrm{m}$ in domain size on the substrates. Occasionally, holes with diameters of 5-20 $\mu \mathrm{m}$ of empty space were found between the domains. Though dip-drying is one of the more common techniques for NSL [8], we found that spin-coating was a more controllable process, especially for patterning a monolayer of beads on wafer-scale substrates.

The tailoring of the size of polystyrene bead masks by oxygen RIE shrunk the diameters of these beads from 500 to $250 \mathrm{~nm}$ linearly with etch time (figure 2, Row A \& figure $3)$. Since the position of each bead remained the same after the oxygen RIE, the separations between each bead mask increased as the sizes of the beads decreased. Though the etching process caused a minor increase in surface roughening on the etched beads, the beads in general were etched evenly in the lateral dimension within the tested time periods of $0-150$ s. The heights of the beads were also etched gradually during the RIE process. However, RIE runs with $180 \mathrm{~s}$ or longer often resulted in near to total removal of the beads, and thus they were used not in our experiments.

The fabrication of silicon nanopillars from the bead masks of different sizes and separation led to pillars of diameters from 200 to $350 \mathrm{~nm}$ and height of about $400 \mathrm{~nm}-2 \mu \mathrm{m}$ by applying the "Bosch" time-multiplexed silicon etch process. During each of the "Bosch" etch cycles, the exposed silicon areas were first etched with $\mathrm{SF}_{6}$ and then passivated with a polymer formed by $\mathrm{C}_{4} \mathrm{~F}_{8}$. Since the bombardment of the reactive ions was directional and perpendicular to the surface of the substrate in the deep RIE etcher, the bottoms of the etched wells were preferentially etched during the etch phase of the etch cycle. The side walls were etched only minimally due to the parallel directionality of the reactive ions to the side walls and the protection of deposited fluorinated polymers. Consequently, with appropriate etch masks and etch parameters, this etch process had been shown to produce silicon structures with high aspect ratio features of 40 and above [17].

For silicon etch periods shorter than 22 cycles, the fabrication scheme yields short silicon pillars with structures as predicted. After five etch cycles, the diameters of the as-fabricated pillars were similar to the size of the five different bead etch masks used (figure 2, row B and row C). The "Bosch" process was shown to effectively etch the silicon substrate though the gaps between the "bead masks," which are of the order of 20-150 nm. This further demonstrates that the chosen etch process for the fabrication of small-size features is crucial over the ordinary RIE process which had been used to fabricate only low aspect ratio wells and pillars [7,8]. Though the size of the bead mask shrunk about $5-10 \%$ in diameter during the silicon etch, the diameters of the pillars still matched the size of the corresponding masks because the diameter was defined during the beginning of the etch process. Moreover, the structures and arrangements of 'bead masks' remained mostly intact after the silicon etch.

The shapes of the cross sections along the silicon pillars in general conform to the shapes of the bead masks. Along the length of a silicon pillar, small but periodic variations of the pillar diameter indicate the etching and passivation cycles 


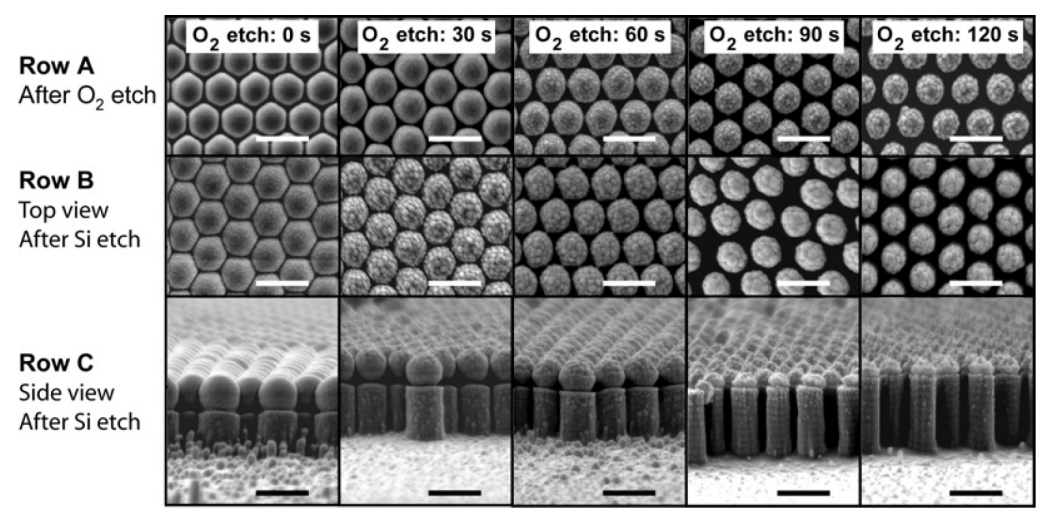

Figure 2. Scanning electron micrographs of nanospheres-on-silicon samples after the second and third step of the nanopillar fabrication scheme as shown in figure 1. Row A: top views of the nanospheres' "resist" on silicon substrates etched by oxygen plasma treatment of periods of 0-120 s. Row B and row C: top and side views of samples in row A etched by deep reactive ion etching using the "Bosch" process for five cycles. All scale bars are 750 $\mathrm{nm}$.

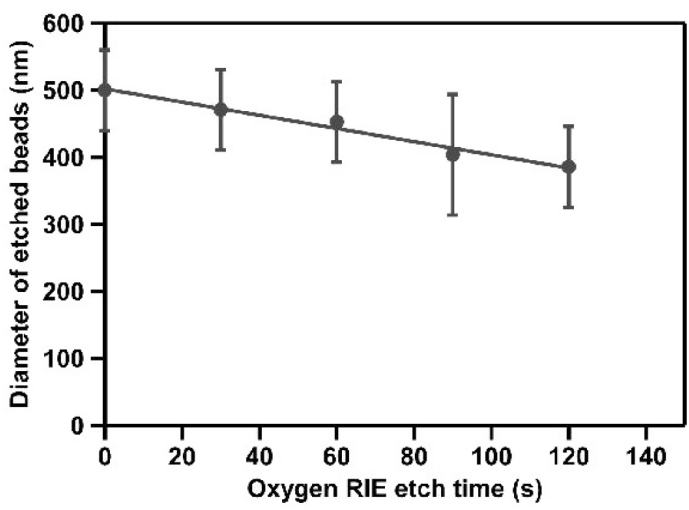

Figure 3. A plot of the diameters of oxygen RIE etched polystyrene beads on silicon substrates versus the RIE etch time. The error bar is \pm 3 standard deviation of each etched bead diameter measurement.

during the "Bosch" process. This "scalloping" effect is usually a function of polymer quantity deposited for etch protection during the passivation period and the strength of the RF power in the etching period.

The etch rate of the silicon substrate was found to be a strong function of the aspect ratio of the etched well geometry. In our case, the etched areas are of a complicated and periodic pattern. To simplify the estimation, we define the aspect ratio as the depth of the etched well (length of the pillar) divided by the average of the minimum separation between the bead masks and ignore the effect of the etch mask pattern on the etch rate. Using the data from figure 2, a graph of silicon substrate etch rates versus the aspect ratio (figure 4)indicates that the silicon etch rate drops as the aspect ratio increases.

The aspect ratio etch rate dependence (ARDE) phenomenon in deep RIE etching is commonly observed in the micrometer and sub-micrometer scales when the aspect ratio of the etched wells is above 1. The ARDE had been extensively studied for trench etching in the $\mu \mathrm{m}$ range; however, little has been reported for high density nanoscale patterns. Various factors such as Knudsen transport of neutrals, ion shadowing and charging effects could contribute to the phenomenon [16]. In our case, since the separation between the bead masks is of the order of $10-150 \mathrm{~nm}$, the transport of

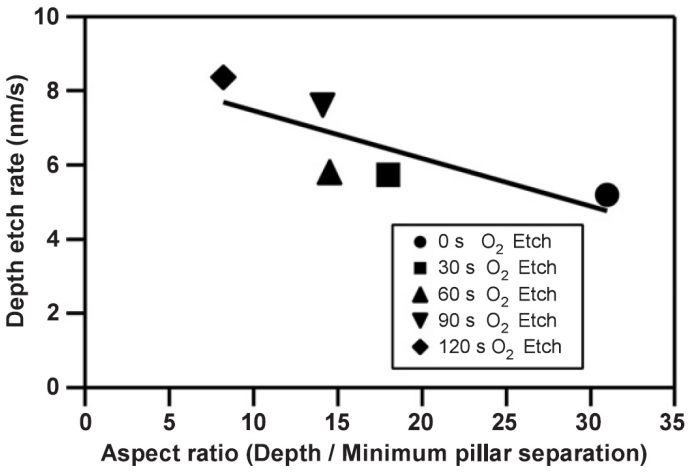

Figure 4. A plot of etch rates of silicon substrates patterned with polystyrene bead masks pre-etched with $0-120$ s oxygen RIE etch periods versus the measured aspect ratio of the corresponding etched wells using the data in figure 2 , row $\mathrm{C}$.

etchants and as-etched gaseous products is the major etch rate limiting factor to etch these pillars. This is further supported by the typical linear relationship between the silicon depth etch rate and the aspect ratio, which is commonly observed in the ARDE analysis for micrometer-scale trench etching experiments limited by chemical transport (Figure 4). Since lower chamber working pressure will increase the mean free path of the molecular species used and produced in the etch process, the etch experiments were carried out at 4.5 mTorr to facilitate the transport and to increase the directionality of these species.

Longer etch progress experiments were also performed to determine the effect of a prolonged etch period on the morphology of the pillar structures and the durability of the bead masks. HCP bead masks on silicon prepared by the above procedure were tailored by oxygen plasma to yield average etched bead diameters of $250 \mathrm{~nm}$ and minimum bead separations of $250 \mathrm{~nm}$. Both 15 and 22 cycles of the silicon "Bosch" etch process were used to etch these samples to generate pillar structures of different aspect ratio (Figure 5). After 15 cycles of etching, the bead masks remained intact and the diameter of the as-etched pillars is similar to those of the bead masks. However, as the number of etch cycles increases from 15 to 22, the bead masks started to degrade. The tops of the 


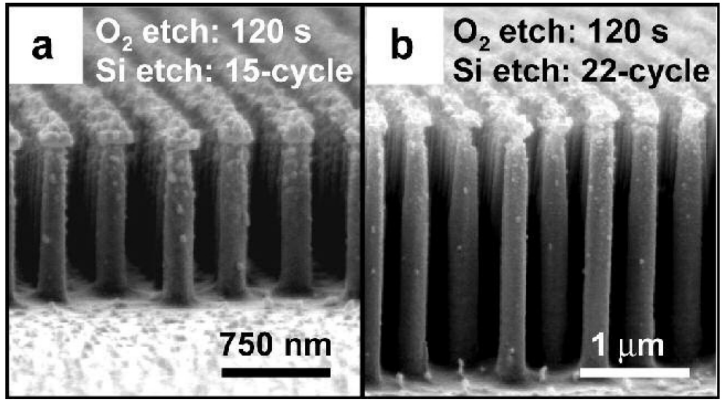

Figure 5. Scanning electron micrographs of nanopillars fabricated from a polystyrene bead patterned silicon surface pre-etched with a 120 seconds oxygen RIE etch period, followed by (a) 15 or (b) 22 cycles of the silicon etch process.

pillars were also etched $5-10 \%$ more due to their better accessibility to etchants in the process. Nevertheless, nearly uniform silicon pillars of about 5 and 10 aspect ratio can be fabricated using the present process parameters without further optimization (Figure 5). Moreover, as the etch time increases, the side walls of the as-etched pillars become smoother without the "scallop features," probably due to a local heating effect in the etching process. The tapering effect at the bottom of the etched areas is found to be minimal. The degrading of the polystyrene beads during the processing limits the uniformity of the as-fabricated pillars. We are currently exploring the use of more robust beads made of cross-linked polymer and silica and optimized etching conditions to make forest of pillars with higher aspect ratios.

\section{Conclusion}

In short, a fabrication scheme that combines nanosphere lithography and reactive ion etching (both standard and deep RIE) was developed to produce hexagonally close packed semiconductor nanopillars. The etch rates of these structures were found to decrease with an increasing aspect ratio and thus the etch process is chemical transport rate limited. Pillars of smaller diameters and of different materials can be potentially fabricated using bead masks of smaller sizes with the present scheme.

Since the patterns generated by nanosphere lithography depend on the geometry of the sphere packing, the reported scheme allowed only a single layer of patterned features to be generated on a substrate at a time. Also, the presented procedure yields only hexagonally close packed pillar patterns of limited domain size with diameters of tens of microns. However, applications which do not require perfect hexagonal patterns such as adhesion growth substrates for cell growth [12] and the production of superhydrophobic surfaces [13] can be envisaged with further chemical functionalization of the pillar surface. Moreover, epitaxially grown semiconductor wafers with different semiconductor layers can be used directly to fabricate nanopillars with self-aligned and well-defined homo or hetero junction properties. Such structures can be used for investigating proof of principle device architecture such as the vertical surround-gate field-effect transistors [18]. Unlike other gas phase nanowire synthesis, our process does not require the patterning of a metal catalyst and thus minimizes the potential for unintentional doping of the wires.

The challenge to generate a large domain size of packed nanospheres depends on the control of the phase of colloidal packing in the solution media and the interaction between the spheres and the substrates. Recently, Jiang et al have reported their progress in generation of nearly single-domain HCP nanosphere patterns on 4 inch wafers by adjusting the viscosity of the silica nanosphere solution with appropriate polymer content [7]. This provides a possible route to extend the limitation of the present procedure to generate nanopillar patterns with longer range order. Hence, the present demonstrated scheme provides an alternative inexpensive method for nanoscale, self-aligned pillar fabrication.

\section{Acknowledgments}

The authors are grateful for the support of the Laboratory Directed Research and Development grant from the Lawrence Livermore National Laboratory (grant \# 04-ERD-107). CLC thanks the support of the start up research fund from the University of Nebraska-Lincoln. This work was performed under the auspices of the US Department of Energy by the University of California, Lawrence Livermore National Laboratory under Contract No. W-7405-Eng-48.

\section{References}

[1] Khann K V 2004 Physics of carrier-transport mechanisms and ultra-small scale phenomena for theoretical modelling of nanometer MOS transistors from diffusive to ballistic regimes of operation Phys. Rep. 398 67-131

[2] Yakushiji Y, Ernult F, Imanura H, Mitani S, Takanashi K, Takahashi S, Maekawa S and Fujimori H 2004 Enhanced spin accumulation and novel magneto transport in nanoparticles Nat. Mater. 4 57-61

[3] Van Blaaderen A 1998 Materials science-Opals in a new light Science 282 887-8

[4] Ormonde A D, Hicks E C, Castillo J and Van Duyne R P 2004 Nanosphere lithography: fabrication of large area Ag nanoparticle arrays by convective self-assembly and their characterization by scanning UV-vis extinction spectroscopy Langmuir 20 6927-31

[5] Albrecht M, Hu G, Guhr I L, Ulbrich T C, Boneberg J, Leiderer $P$, and Schatz G 2005 Magnetic multilayers on nanospheres Nat. Mater. 4 203-6

[6] Whitney A V, Myers B D, and Van Duyne R P 2004 Sub-100 nm triangular nanopores fabricated with the reactive ion etching variant of nanosphere lithography and angle-resolved nanosphere lithography Nano Lett. 4 1507-11

[7] Jiang P and McFarland M J 2005 Wafer-scale periodic nanohole arrays templated from two-dimensional nonclose-packed colloidal crystals J. Am. Chem. Soc. 127 3710-1

[8] Kuo C, Shiu J and Chen P 2003 Sub-10 nm fabrication of large-area periodic nanopillar Mater. Res. Soc. Symp. Proc. EXS-2 M5.11.1-11.13

[9] Rose J and Baugh D 2004 Nanorings, nanopillars and nanospikes on $\mathrm{Si}(111)$ by modified nanosphere lithography: fabrication and application Mater. Res. Soc. Symp. Proc. 832 F7.14.1-14.11 
[10] Kuo C-W, Shiu J-Y, Chen P L and Somorjai G A 2003 Fabrication of size-tunable large-area periodic silicon nanopillar arrays with sub-10-nm resolution J. Phys. Chem. B 107 9950-3

[11] Kaji N, Tezuka Y, Takamura Y, Ueda M, Nishimoto T, Nakanishi H, Horiike Y and Baba Y 2004 Separation of long DNA molecules by quartz nanopillar chips under a direct current electric field Anal. Chem. 76 15-22

[12] Kim P, Kim D H, Kim B, Choi S K, Lee S H, Khademhosseini A, Langer R, and Suh K Y 2005 Fabrication of nanostructures of polyethylene glycol for applications to protein adsorption and cell adhesion Nanotechnology 16 2420-6

[13] Martines E, Seunarine K, Morgan H, Geadegaard N, Wilkinson C D W, and Riehle M O 2005 Superhydrophobicity and superhydrophilicity of regular nanopatterns Nano Lett. 5 2097-103
[14] Hulteen C and Van Duyne R P 1995 Nanosphere lithography: a materials general fabrication process for periodic particle array surfaces J. Vac. Sci. Technol. A 13 1553-8

[15] Blauw M A, Zijlstra T, and van der Drift E 2001 Balancing the etching and passivation in time-multiplexed deep dry etching of silicon J. Vac. Sci. Technol. B 19 2930-4

[16] Gottscho R A, Jurgensen C W, and Vitkavage D J 1992 Microscopic uniformity in plasma-etching J. Vac. Sci. Technol. B $102133-47$

[17] Ayazi F and Najafi K 2000 High aspect-ratio polysilicon micromachining technology Sensors Actuators 87 46-51

[18] Ng H T, Han J, Yamada T, Nguyen P, Chen Y P, and Meyyapan M 2004 Single crystal nanowire vertical surroundgate field-effect transistor Nano Lett. 4 1247-52 